\section{PTU-097 A 'REAL-LIFE' PICTURE OF ABNORMAL LIVER FUNCTION TEST MANAGEMENT IN PRIMARY CARE IN HULL, EAST YORKSHIRE}

doi:10.1136/gutjnl-2013-304907.187

1."B Williams, 'G Abouda, ${ }^{2}$ M Follows. ' Gastroenterology Research Department, Hull and East Yorkshire Hospitals NHS Trust; ${ }^{2}$ GP, NHS Hull, Hull, UK

Introduction As part of a community based pilot study looking at NAFLD and Enhanced Liver Fibrosis (ELF) testing (COMMANDS), an audit of GP management of Abnormal Liver Function (LFT) tests was conducted. The aim was to gain a greater understanding of 'real-life' community based clinical management of liver disease.

Methods The audit consisted of a retrospective SYSTM 1 database search $(2009$ - 2011) for patients with an ALT $\geq 70$, from 6 GP practises in NHS Hull. Data from 162 patients was collected and results were analysed using the COMMANDS GP liver screen as the acceptable standard of practise, a combination of liver disease risk factors and investigations.

Results Gender distribution was 111 men to 51 women; the average age was 49 years.

Risk factors for liver disease were under-reported as shown below.

Graph 1 - Percentage of Patients in Primary Care with Documented Risk Factors

Investigations for liver disease revealed that patients were not adequately screened.

Graph 2 - Percentage of Patients in Primary Care with Documented Investigations

Only $18 \%$ of patients had a documented diagnosis. GPs gave a diagnosis in $11 \%$ of cases and $7 \%$ were referred to a specialist.

Graph 3 - Referral Pattern for Patients with a Documented Diagnosis

We collected up to 10 retrospective abnormal ALT results per patient working back from the data collection date. In total, we recorded 562 abnormal ALT results.
Graph 4 - Distribution of ALT Results in Audit Patients

GP actions taken to manage the abnormal ALT results above, as defined by SYSTM1 drop down boxes, were reviewed. The audit found that ALT results were often incorrectly assessed by GPs according to standard laboratory parameters.

Graph 5-GP and Audit Labelling of ALT Results

Conclusion This audit showed that there is a lack of consistency in how GPs make clinical decisions. There is no clearly defined standard practise for investigating abnormal LFTs in primary care. More work on management guidelines that are easy and practical is required.

Disclosure of Interest None Declared

\section{PTU-098 A SURVEY TO INVESTIGATE GP KNOWLEDGE, PRACTICE AND SUPPORT REQUIREMENTS REGARDING THE CLINICAL MANAGEMENT OF LIVER DISEASE IN PRIMARY CARE}

doi:10.1136/gutjnl-2013-304907.188

1,"B Williams, 'G Abouda. 'Gastroenterology Research Department, Hull and East Yorkshire Hospitals NHS Trust, Hull, UK

Introduction The management of liver disease in primary care often lacks consistency and clearly defined care pathways. We aimed to explore GP perspectives on the management of liver disease in the Hull and East Riding of Yorkshire region.

Methods A 9 question survey was sent to 321 GPs in Hull and East Riding of Yorkshire focusing on knowledge, practise and support requirements of liver disease management in primary care. 105 GPs (33\%) responded: $26 \%$ from NHS Hull and 39\% from NHS East Riding Results GPs saw patients with a variety of liver diseases. Most common were lifestyle-related.

Graph 1 - Liver diseases seen by GPs very often, often or sometimes

GPs graded the importance of a range of liver investigations in primary care.
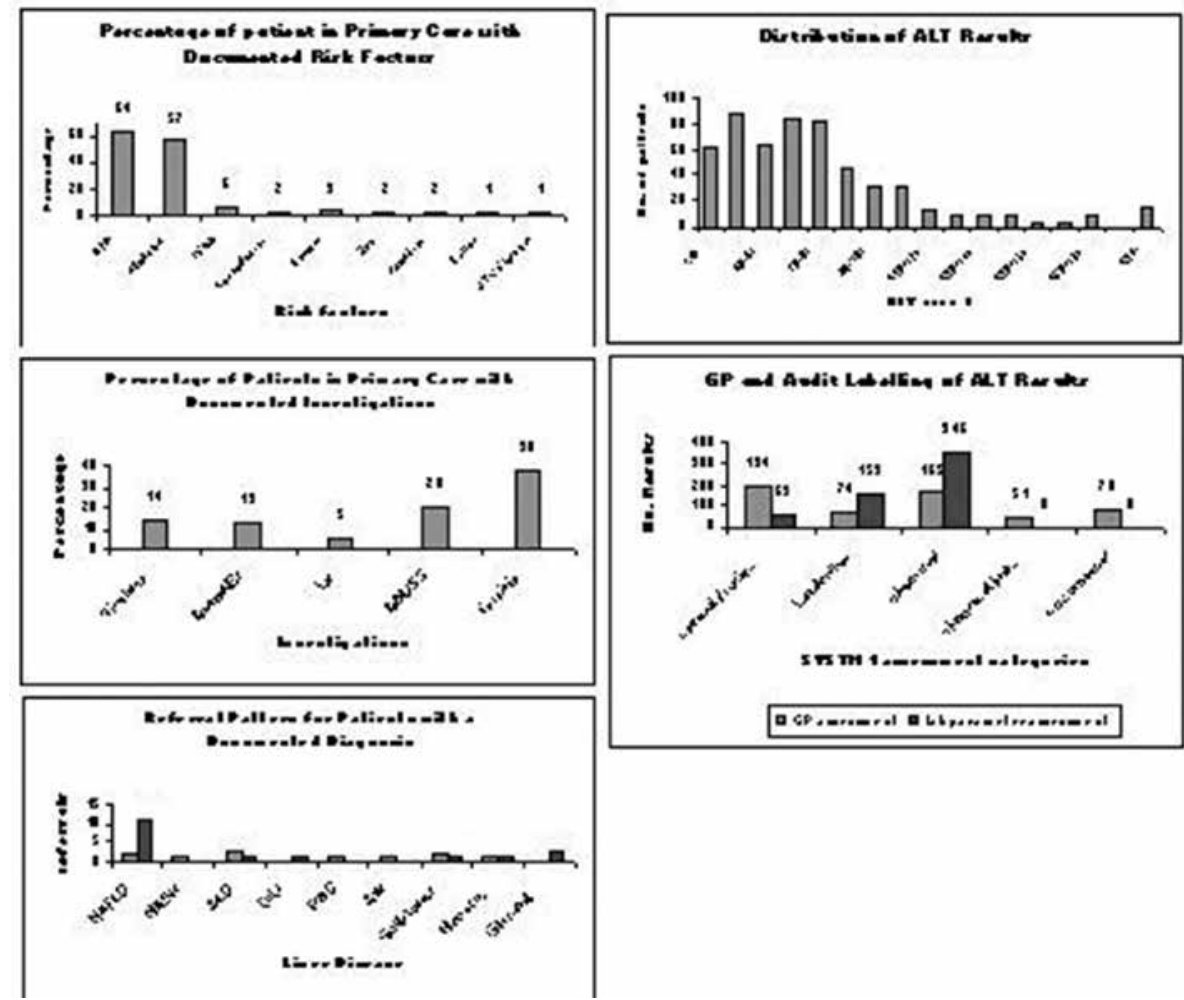

Abstract PTU-097 Figure 


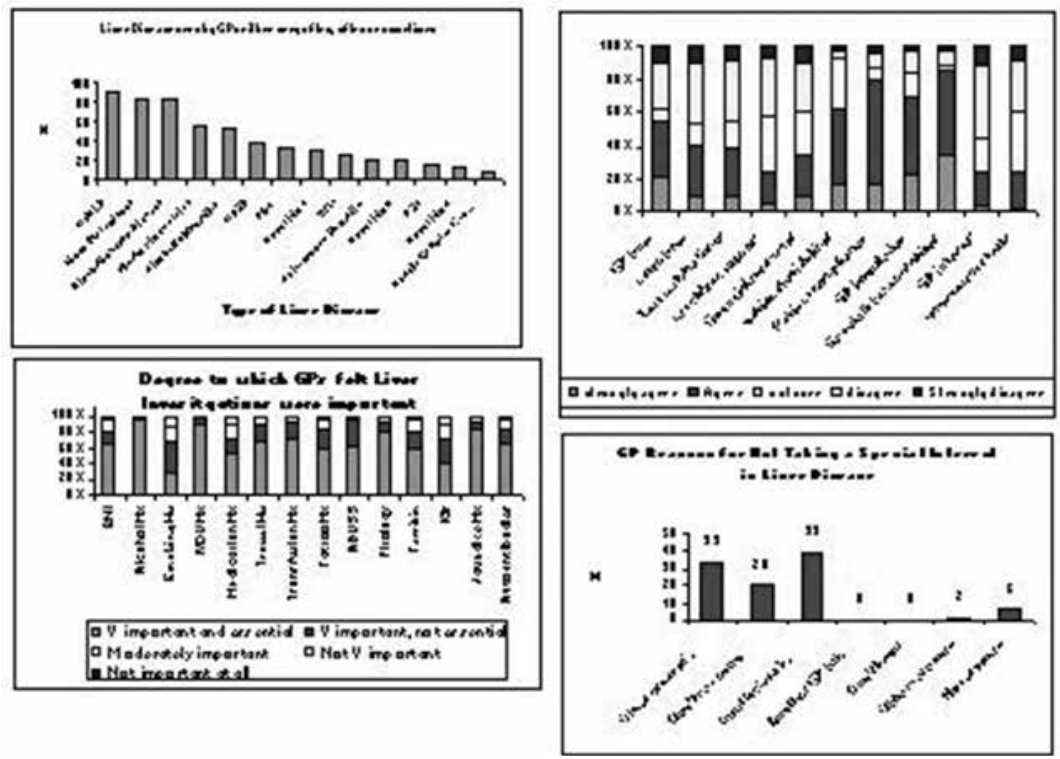

Abstract PTU-098 Figure

Graph 2 - The degree to which GPs felt liver investigations were important in the clinical management of liver disease in primary care.

For investigations that GPs graded as 'Very Important and Essential' many were not, in 'real-life' primary care practise, documented in patient records (Williams et. al. 2012).

Graph 3 - The degree to which potential barriers might influence GP capacity to manage liver disease in primary care.

Only $6 \%$ of GPs stated they had a 'special interest' in liver disease and no GP stated that someone else in the practise took the lead on liver disease.

Graph 4 - Reasons for GPs not having a 'special interest' in liver disease

However, as 'generalists', 83\% of GPs felt they needed more educational support via protected learning sessions, improved national guidelines and joint specialist-GP clinics.

Conclusion This survey revealed that many GPs, despite not having a 'special interest' in liver disease, would welcome greater educational support from specialists and improved national guidelines. The barriers most cited as influencing GP capacity to manage liver disease are surmountable and should be the focal point for new integrated care pathways.

Disclosure of Interest None Declared

\section{PTU-099 DISCOVERY OF POTENTIAL PLASMA BIOMARKERS OF CHOLANGIOCARCINOMA UTILISING SURFACE-ENHANCED LASER DESORPTION/IONIZATION TIME-OF-FLIGHT MASS SPECTROMETRY (SELDI-TOF MS)}

doi:10.1136/gutjnl-2013-304907.189

1."C A Wadsworth, 2V M Horneffer-van der Sluis, 'A Zabron, 'S D Taylor-Robinson, 'R J Edwards, 'S A Khan. 'Department of Hepatology and Gastroenterology; ${ }^{2}$ Centre of Pharmacology and Therapeutics, Imperial College London, London, UK

Introduction Cholangiocarcinoma (CC) is a malignant neoplasm of the bile duct. Diagnosis of CC is hampered by the inadequate performance of current plasma markers of disease, particularly in patients with preexisting primary sclerosing cholangitis (PSC). We aimed to identify potential new protein biomarkers of CC

Methods In an initial discovery study, blood plasma samples from 18 subjects with CC, 17 with PSC and 10 healthy controls were subjected to SELDI-TOF MS. Comparisons of $\mathrm{m} / \mathrm{z}$ peak intensity were made between groups using the Mann-Whitney $U$ test. Differentiating $\mathrm{m} / \mathrm{z}$ peaks were then confirmed in a further validation study of 81 subjects with CC, 54 with PSC and 90 healthy controls. Pearson's correlation was used to investigate the relationship of each $\mathrm{m} / \mathrm{z}$ peak's intensity to routine laboratory indices. Diagnostic performance was investigated using receiver operator characteristic area-under-the curve (ROC-AUC) analyses. Multiple linear regression was used to investigate the performance of combinations of differentiating $\mathrm{m} / \mathrm{z}$ peaks, as well as the combination of $\mathrm{m} / \mathrm{z}$ peaks with routine laboratory markers (including CA19-9).

Results Seven differentially expressed $\mathrm{m} / \mathrm{z}$ peaks were identified in the CC group and these were subsequently confirmed in the validation study $(p=2.6 \times 10-4$ to $9.4 \times 10-13)$. The intensity of the seven $\mathrm{m} / \mathrm{z}$ peaks of interest did not correlate with creatinine, ALP, bilirubin, CRP, white cell count or CA19-9. A panel of three peaks discriminated CC from PSC subjects with ROC-AUC of 0.76 (sensitivity $75 \%$, specificity $64 \%$ ). A panel of five peaks discriminated CC subjects from healthy controls with ROC-AUC of 0.90 (sensitivity $95 \%$, specificity $74 \%$ ). Addition of routine laboratory indices did not change the diagnostic performance of these models significantly.

Conclusion SELDI-TOF has been used to successfully identify seven $\mathrm{m} / \mathrm{z}$ peaks that are differentially intense in CC subjects (total $\mathrm{n}=99)$, when compared to PSC subjects $(\mathrm{n}=64)$ and healthy controls $(n=107)$. These peaks appear to be independent of standard markers of renal impairment, cholestasis, sepsis and inflammation, as well as CA19-9. Individually, and more so in combination, these peaks exceed the expected diagnostic performance of CA19-9, particularly in discriminating CC from PSC. Work to identify the proteins represented by these $\mathrm{m} / \mathrm{z}$ peaks is ongoing.

Disclosure of Interest None Declared

\section{PTU-100 DECOMPENSATED ALCOHOLIC LIVER DISEASE (ALD) IS ASSOCIATED WITH STARTING HEAVY DRINKING AT AN OLDER AGE: A CASE-CONTROL STUDY}

doi:10.1136/gutjnl-2013-304907.190

1,"D Gleeson, 'A K Ali, ' J S Jones, ' M Bradley, ${ }^{2} \mathrm{R}$ J Peck, ${ }^{3} \mathrm{~K}$ M McCormack. 'Liver Unit; ${ }^{2}$ Radiology; ${ }^{3}$ Research, Sheffield Teaching Hospitals, Sheffield, UK 